\title{
Is Nasal Polyp in Pediatric Cases Same as in Adult Population?
}

\author{
Shyam Sudhakar Sudarsan ${ }^{1}$, Indu Rajkumar ${ }^{2}$, Revathi Shree $\mathrm{K}^{3}$, Srinivasan $\mathrm{K}^{4}$
}

\begin{abstract}
Nasal polyposis is a chronic inflammatory disease affecting the nasal cavity and paranasal sinuses. Nasal polyps represent the end-stage local manifestation of chronic inflammatory disease of the sinonasal tract. Morphologically, nasal polyps are edematous grape-like protrusions most often originating in the upper part of the nose around the osteomeatal complex (OMC) on the lateral wall. Polyps can vary widely in size and should be considered a bilateral condition. Aim of our study was to evaluate the characteristics and clinical features of nasal polyps in pediatric and adult population.

Medical records of 232 patients (11 children and 221 adults) evaluated nasal polyps between 2015 and 2018 were reviewed retrospectively. Demographic characteristics, clinical presentation, management and histological findings were compared of the 232 patients, 11 were children and 221 were adults. Nasal obstruction was the most common presenting symptom in both groups. The incidence of snoring was more common among the pediatric age group as compared to the adult group and was found to be statistically significant. Epistaxis was also found to be more common among the pediatric age group, while sinusitis was noted to be significantly more common among the adult group. On histologic examination, allergic nasal polyp was more common than inflammatory nasal polyp in children as compared to adults.

Conclusion: Nasal polyps are more common in adults. Children have unique clinical features such as increased snoring and epistaxis and predominant allergic histology as compared to adults warranting special attention in devising management plan.

Keywords: Adult polyps, Antrochoanal, Epistaxis, Ethmoidal, Functional endoscopic sinus surgery, Nasal polyposis, Pediatric polyps, Nasal obstruction, Osteomeatal complex.

International Journal of Head and Neck Surgery (2021): 10.5005/jp-journals-10001-1451
\end{abstract}

\section{INTRODUCTION}

Nasal polyposis is a chronic inflammatory disease affecting the nasal cavity and paranasal sinuses. Nasal polyps represent the end-stage local manifestation of chronic inflammatory disease of the sinonasal tract. European guidelines ${ }^{1}$ define nasal polyps clinically as inflammation of the nose and paranasal sinuses associated with two or more symptoms, one of which should be nasal blockage/obstruction/congestion or nasal discharge, \pm facial pain/pressure, reduction or loss of smell and either endoscopic evidence of polyps and/or mucopurulent discharge from the middle meatus or edema, mucosal obstruction primarily in the middle meatus and/or computed tomography (CT) changes such as mucosal changes within the osteomeatal complex and/or sinuses.

Morphologically, nasal polyps are edematous grape-like protrusions most often originating in the upper part of the nose around the osteomeatal complex on the lateral wall. The surface epithelium tends to be smooth and consists of pale translucent tissue, which distinguishes them from the more vascular mucosa of the nasal cavity. Polyps can vary widely in size and should be considered a bilateral condition. It is a relatively common disease occurring in $1-4 \%$ of the adult population, ${ }^{2}$ but it is less commonly described in pediatric population. Most of the published series include children presenting with other underlying systemic diseases, mainly cystic fibrosis.

\section{Aim and Objective}

To describe the characteristics of pediatric patients suffering from nasal polyposis and compare it with that of adult population.

\section{Materials and Methods}

This was a retrospective study conducted at the Department of Otorhinolaryngology of a tertiary care hospital. The study was

\begin{abstract}
${ }^{1-4}$ Department of Otorhinolaryngology, Saveetha Medical College and Hospital, Thandalam, Chennai, Tamil Nadu, India

Corresponding Author: Shyam Sudhakar Sudarsan, Department of Otorhinolaryngology, Saveetha Medical College, Thandalam, Chennai, Tamil Nadu, India, e-mail: b7shyam@yahoo.com
\end{abstract}

How to cite this article: Sudarsan SS, Rajkumar I, K RS, et al. Is Nasal Polyp in Pediatric Cases Same as in Adult Population? Int J Head Neck Surg 2021;12(3):98-100.

Source of support: Nil

Conflict of interest: None

conducted according to the Declaration of Helsinki. Clearance for the study was obtained from the Institutional Review Board and the Ethics Committee of our institution. Written informed consent was not obtained due to retrospective nature of the study. The medical records of all patients diagnosed with nasal polyps, treated and followed-up between June, 2018 and May, 2019 in our Department of Otorhinolaryngology were reviewed. The demographics, clinical presentation, treatment, histology, and outcomes were recorded (Table 1, Figs 1 to 3 ). The study population was then subdivided into pediatric ( $\leq 18$ years of age) and adult ( $>18$ years of age) groups for statistical analysis. The groups were matched in all aspects, except age. All patients underwent diagnostic nasal endoscopy (DNE) and CT scans of the paranasal sinuses in coronal, axial, and sagittal planes. Magnetic resonance imaging (MRI), if needed, was done. Nasal endoscopy and CT are excellent tools for diagnosing nasal polyps. ${ }^{3}$ Preoperatively, routine blood investigations were done and anesthetic fitness for surgery was obtained. Statistical tool used-IBM SPSS v22data were represented as number (percentage). Unpaired t-test was done to evaluate statistical significance. $p$ value $<0.05$ was considered statistically significant.

(O)The Author(s). 2021 Open Access This article is distributed under the terms of the Creative Commons Attribution 4.0 International License (https://creativecommons. org/licenses/by-nc/4.0/), which permits unrestricted use, distribution, and non-commercial reproduction in any medium, provided you give appropriate credit to the original author(s) and the source, provide a link to the Creative Commons license, and indicate if changes were made. The Creative Commons Public Domain Dedication waiver (http://creativecommons.org/publicdomain/zero/1.0/) applies to the data made available in this article, unless otherwise stated. 
Table 1: Demographic details

\begin{tabular}{llll}
\hline Parameter & Pediatric $n=11$ & Adult $n=221$ & $p$ value \\
\hline Male & $7(63.6 \%)$ & $145(65.6 \%)$ & 0.001 \\
Female & $4(36.4 \%)$ & $76(34.4 \%)$ & 0.001 \\
Nasal obstruction & $11(100 \%)$ & $206(93.2 \%)$ & 0.001 \\
Nasal discharge & $9(81.8 \%)$ & $184(83.2 \%)$ & 0.001 \\
Allergy & $4(36.3 \%)$ & $36(16.2 \%)$ & 0.001 \\
Epistaxis & $8(72.7 \%)$ & $124(56.1 \%)$ & 0.001 \\
Sinusitis & $10(90.9 \%)$ & $128(57.9 \%)$ & 0.001 \\
Headache & $3(27.2 \%)$ & $192(86.8 \%)$ & \\
\hline
\end{tabular}

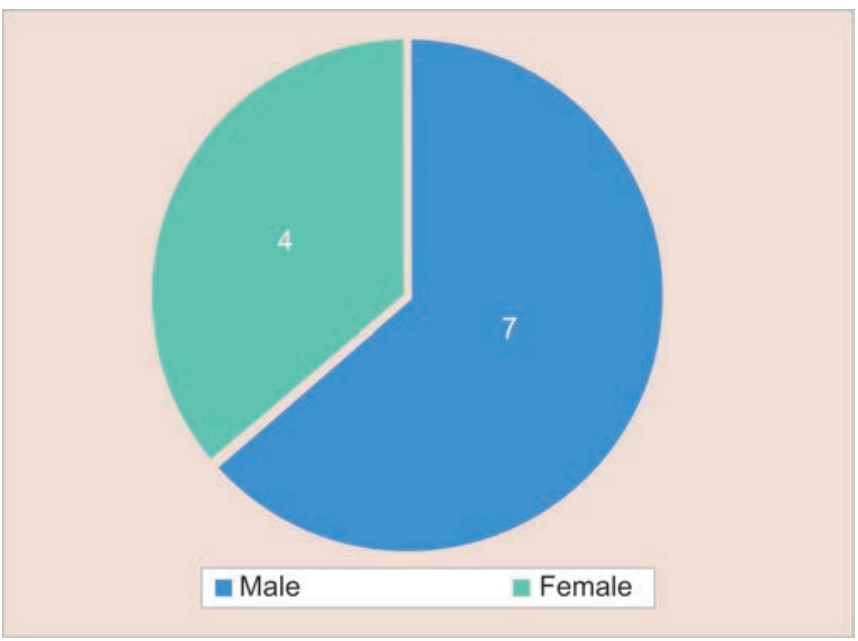

Fig. 1: Pediatric gender distribution

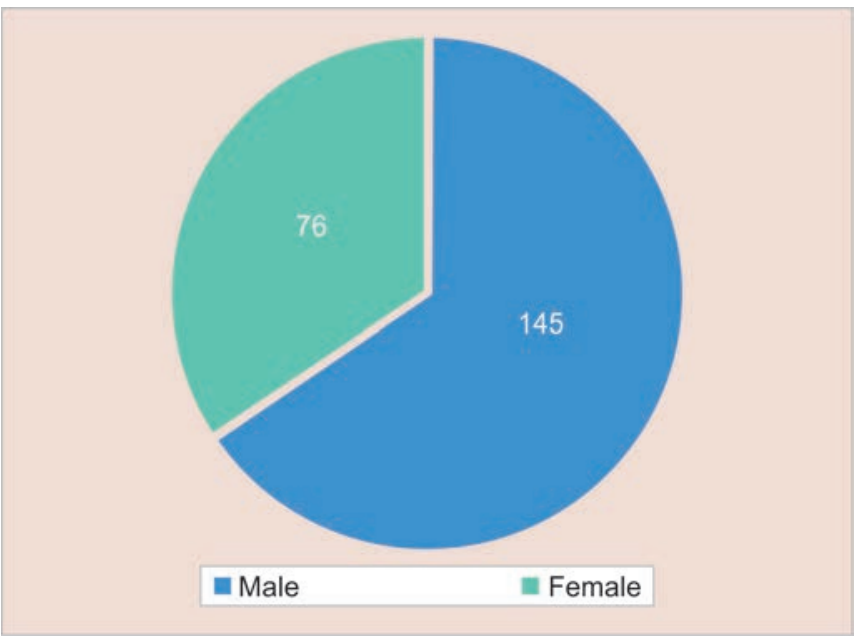

Fig. 2: Adult gender distribution

Surgery was performed under general anesthesia after written informed consent. The first stage was the removal of the intranasal portion of the polyp, usually with microdebrider to identify the origin of the lesion from the middle meatus. Following this, a partial inferior uncinectomy and a middle meatal antrostomy were performed. In adult patients, inferior turbinectomy and uncinectomy followed by middle meatal antrostomy were done. Frontoethmoidectomy and sphenoidotomy were done if involved. Nasal packing was done immediately after surgery and was retained up to 24-48 hours after surgery.

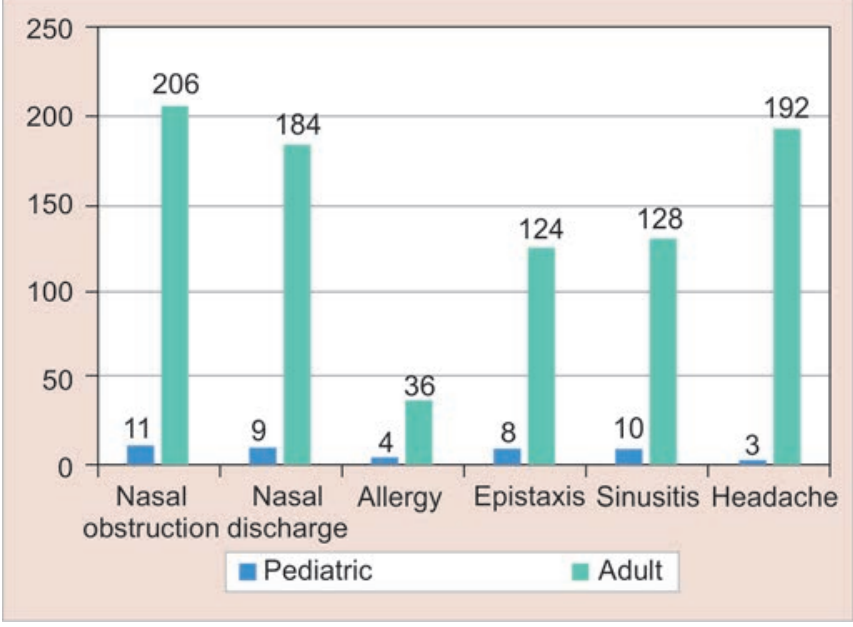

Fig. 3: Type of presentation in pediatric and adult population

After the surgical removal of nasal polyps, all excised lesions were histopathologically studied (hematoxylin and eosin staining) by the pathologist to confirm the diagnosis and also to evaluate potential histological characteristics of nasal polyps in adults and children. All patients received broad-spectrum antibiotic therapy for 1 week after surgery. Saline irrigations were recommended until the nasal mucosa completely healed. Patients were usually discharged after 4 or 5 days postoperatively. The first endoscopic consultation was usually performed 1 week postoperatively. Subsequent follow-up endoscopic examinations (using $0^{\circ}$ and $45^{\circ}$ rigid nasal scopes) were set at 1, 3, 6 months and 1 year after surgery, and then annually in order to detect recurrences.

\section{Results and Discussion}

A total of 232 patients were included in this study out of which 221 belonged to adult population and 11 patients belonged to pediatric population. In adult population, male:female ratio was 1.9:1 with male preponderance. In pediatric population also, male:female ratio was 1.7:1 with male preponderance. In pediatric population, all 11 patients were found to have antrochoanal polyps; whereas, in adult population, 175 patients had ethmoidal polyps and 46 patients had antrochoanal polyps (Table 2). In a similar study done by Aleksandar Perić et al., 78 patients with choanal polyps were included out of which 22 (28\%) patients were children and 56 (72\%) patients were adults. Antrochoanal polyps are found more frequently in children than adults and represent up to $42 \%$ of all nasal polyps in children and $4-6 \%$ in adults. ${ }^{5-7}$

The most common presenting complaint in both adult and pediatric population was found to be nasal obstruction 
Table 2: Gross pathology noted in our series

\begin{tabular}{lll}
\hline Gross pathology & Pediatric & Adult \\
\hline Ethmoidal & 0 & 175 \\
Antrochoanal & 11 & 46 \\
Total & 11 & 221 \\
\hline
\end{tabular}

(93.2\% and $100 \%$ in adult and pediatric population, respectively) and it was found to be in accordance with a study conducted by Dong Hoon Lee et al. ${ }^{8} 4$ patients in pediatric population and 36 patients in adult population had some form of documented allergic disorder. Epistaxis was seen in 8 out 11 patients in pediatric population and 124 out of 221 patients in adult population. In a study conducted by Ahmad Meymane Jahromi et al., ${ }^{9} 11.1 \%$ of patients had a history of epistaxis. Sinusitis (as evidenced by imaging-X-ray PNS or CT PNS) was observed in 10 out of 11 patients in pediatric population compared to 128 out of 221 patients in adult population and this difference was found to be statistically significant $(p=0.001)$.

While 3 out of 11 patients in pediatric population had headache, 192 out of 221 patients in adult population complained of headache and this was found to be statistically significant ( $p$ value $<0.01$ ). Surgical removal is the generally accepted treatment of choice for antrochoanal polyps. ${ }^{10-12}$ All of them had the polyps removed by transnasal approach (Functional Endoscopic Sinus Surgery) and submitted for histopathological examination. By histopathological examination, polyps were classified as allergic, if the mucosal surface had respiratory epithelium, absence of mucus glands, and plenty of eosinophils or inflammatory, if mucosal surface had respiratory epithelium, absence of mucosal glands, and plenty of neutrophils. Allergic pattern in histology was more common in pediatric population while inflammatory pattern predominated in the adult population (Table 3 ). This difference was found to be statistically significant ( $p$ value $<0.01$ ). In a study done by Khalid Al-Mazrou et al., ${ }^{6}$ it was also found that allergic polyps (abundant eosinophils) were significantly more common than inflammatory polyps (abundant neutrophils) among children; while in adults, inflammatory polyps were more common than allergic polyps.

\section{Conclusion}

Nasal polyps are more common in adults. The occurrence of this disease in pediatric population raises the question of whether it is the same disease as seen in adults and if the clinical work-up and treatment follow-up should be the same. Children have distinct
Table 3: Histopathological findings in pediatric and adult population

\begin{tabular}{lll}
\hline Histopathology & Pediatric & Adult \\
\hline Allergy & 9 & 190 \\
Inflammatory & 2 & 31 \\
Total & 11 & 221 \\
\hline
\end{tabular}

clinical features such as increased epistaxis and predominant allergic histology as compared to adults warranting special attention in devising management plan.

Ethical compliance: Complied

\section{References}

1. Fokkens WJ, Lund VJ, Mullol J, et al. EPOS: European position paper on rhinosinusitis and nasal polyps 2012 A summary for otorhinolaryngologists. Rhinology 2012;50:1-12. DOI: 10.4193/ Rhino12.000

2. Caimmi D, Matti E, Pelizzo G, et al. Nasal polyposis in children. J Biol Regul Homeost Agents 2012;26: 77-83.

3. Yaman H, Yilmaz S, Karali E, et al. Evaluation and management of antrochoanal polyp. Clin Exp Otorhinolaryngol 2010;3: 110-114. DOI: 10.3342/ceo.2010.3.2.110

4. Perić $A$, Vukadinović $T$, Kujundžić $T$, et al. Choanal polyps in children and adults: 10-year experience from a tertiary care hospital. Eur Arch Otorhinolaryngol 2019;276(1):107-113. DOI: 10.1007/s00405-0185208-9

5. Segal N, Gluk O, Puterman M. Nasal polyps in the pediatric population. B-ENT 2012;8(4):265-267.

6. Frosini P, Picarella G, De Campora E. Antrochoanal polyp: analysis of 200 cases. Acta Otorhinolaryngol Itali 2009;29(1):21-26.

7. Al-Mazrou KA, Bukhari M, Al-Fayez Al. Characteristics of antrochoanal polyps in the pediatric age group. Ann Thorac Med 2009;4(3):133-136. DOI: 10.4103/1817-1737.53353

8. Lee DH, Yoon TM, Lee JK, et al. Difference of antrochoanal polyp between children and adults Int J Pediatr Otorhinolaryngol 2016;84:143-146. DOI: 10.1016/j.ijporl.2016.03.004

9. Jahromi MA, Pour SA. The epidemiological and clinical aspects of nasal polyps that require surgery. Iran J Otorhinolaryngol 2012;24(67):75-78.

10. El-Sharkawy AA. Endoscopic management of paediatric antrochoanal polyp: our experience. Acta Otorhinolaryngol Ital 2013;33:107-111.

11. Lee TJ, Huang SF. Endoscopic sinus surgery for antrochoanal polyps in children. Otolaryngol Head NeckHead 2006;135:688-692. DOI: 10.1016/j.otohns.2006.02.035

12. Nour YA. Variable extent of nasonantral window for resection of antrochoanal polyp: selection of the optimum endoscopic approach. Eur Arch Otorhinolaryngol 2014;272:1127-1134. DOI: 10.1007/s00405-014-3178-0 